\title{
Iberoamérica en los currículos autonómicos de Ciencias Sociales de Educación Primaria: ¿realidad educativa o declaración de intenciones?
}

\section{Ibero-America in the Spanish curricula of Social Sciences in Primary Education: educational reality or declaration of intent?}

\section{Delfín Ortega Sánchez ${ }^{(1)}$ Francisco Rodríguez Lestegás ${ }^{(2)}$}

(1) Universidad de Burgos (2) Universidad de Santiago de Compostela

Resumen: El presente estudio pretende aproximarse a la determinación curricular de la dimensión iberoamericana, así como a sus implicaciones culturales identitarias, mediante el análisis de los planteamientos y enfoques prescritos en las nuevas disposiciones autonómicas de la LOMCE para la Educación Primaria en España. Asimismo, revisa la presencia y potencial adopción de perspectivas interculturales en la enseñanza de la Historia, y específicamente de la iberoamericana, para este nivel educativo.

Palabras clave: Iberoamérica, enseñanza de la Historia, interculturalidad, diversidad cultural, Educación Primaria.

Abstract: This study analyzes the Ibero-American supranational reality and its cultural implications of a possible common cultural identity. With this intention, we analyze the approaches and expositions of the new educational reform in Spain (LOMCE) in Primary Education. In this respect, the investigation checks the intercultural perspectives in the history education, and of Ibero-America, in the curriculum for this educational level.

Keywords: Ibero-America, History Teaching, interculturality, cultural diversity, primary education.

(Fecha de recepción: febrero, 2016, y de aceptación: mayo, 2016)

DOI: 10.7203/DCES.31.7946 


\section{De la Carta Cultural \\ Iberoamericana al Espacio \\ Cultural Iberoamericano}

La contribución a la consolidación de la identidad iberoamericana, construida sobre la base de su diversidad y multiplicidad cultural, y el fomento de la educación intercultural en la promoción de la identidad de los pueblos iberoamericanos, son dos de los objetivos y fines mejor definidos de la Organización de Estados Iberoamericanos para la Educación, la Ciencia y la Cultura (OEI). En efecto, la Comunidad Iberoamericana, a través de la celebración de sus Cumbres, ha venido visibilizando la proyección global de la región y manifestando su preocupación por el reconocimiento de una identidad cultural iberoamericana con capacidad para la protección de la diversidad cultural que la singulariza. En este sentido, la Carta Cultural Iberoamericana, adoptada en la XVI Cumbre de Jefes de Estado y de Gobierno (Montevideo, 2006), se desarrolla en la apuesta de una conciencia cultural común, capaz de hacer converger la diversidad de expresiones y manifestaciones culturales que la definen. A partir de su riqueza cultural, y entendiendo la idea de Iberoamérica como "un gran sistema donde aparecen elementos únicos y excepcionales" y como "poseedora de un patrimonio cultural común y diverso que es indispensable promover y proteger" (Carta Cultural Iberoamericana, 2006), la declaración supuso el acicate para la consolidación del Espacio Cultural Iberoamericano y su posición, sin- gular y concreta, en un marco de acción universal globalizado.

Desde su ámbito de aplicación "Cultura y Educación”, la Carta prescribe, entre otras líneas de actuación, el refuerzo, en el seno de los sistemas educativos, del conocimiento y valoración de la diversidad cultural iberoamericana, y la incorporación en las propuestas curriculares de las naciones de la comunidad de contenidos propios de la historia y cultura iberoamericanas, "reafirmando sus componentes propios e identitarios" y fomentando "una perspectiva regional del aprendizaje" (Carta Cultural Iberoamericana, 2006). En suma, las bases para la configuración de una dimensión iberoamericana y su inclusión en el ámbito educativo.

La atención sobre los contenidos curriculares iberoamericanos fue concretada en la Cátedra de Historia de Iberoamérica de la OEI desde 1999, con la finalidad de promover e incorporar herramientas y contenidos específicos sobre los procesos históricos propios de los países iberoamericanos; una finalidad ya concretada en el proyecto de la OEI para la Armonización e innovación de la enseñanza de la Historia en Iberoamérica en 1993, y apuntada en la Declaración Viña del Mar (Chile) en 1996.

\section{Hacia la conformación de una dimensión iberoamericana desde la enseñanza de la Historia}

Los textos escolares, concreciones curriculares de las normativas vigentes, tradicionalmente han dado cuenta de la 
presencia de los países de Iberoamérica, con mayor o menor grado de intensidad (Valls, 2012), y con un carácter propagandístico y variable en la construcción de relatos nacionales, estereotipados y excluyentes hasta tiempos recientes. De hecho, puede afirmarse que la adopción de enfoques interculturales ha irrumpido en el ámbito educativo, y específicamente en el propio de la enseñanza de la Geografía y de la Historia, con relativa actualidad.

De forma progresiva, la formación de ciudadanos en torno a la nación y a la configuración de identidades nacionales -una de las clásicas finalidades de la enseñanza de las Ciencias Sociales-, afortunadamente, ha venido desdibujándose para dar lugar a concepciones supranacionales como la europea, la iberoamericana o la mediterránea (Valls, 2011), en las que la interculturalidad se presenta como uno de sus fundamentos obligados. Desde esta perspectiva, la consolidación de identidades nacionales ha visto reducida también su funcionalidad territorial-fronteriza determinista para impulsar una idea de identidad en plural, heterogénea, compleja, inclusiva y multicultural. En esta línea, y de acuerdo a la Carta Cultural Iberoamericana y al reconocimiento del Espacio Cultural Iberoamericano, la definición de la dimensión iberoamericana no ha de ser abordada ni entendida desde un supuesto consenso cultural, en un intento por reducir al común denominador territorios que ahora se amplían.

La diversidad cultural, presente como elemento constituyente de las sociedades actuales y como uno de los principios de las políticas culturales de la Organización de los Estados Iberoamericanos, ha venido ocupando un lugar de primer orden en el ámbito educativo. La escuela, en tanto espacio para la transformación y el cambio social, asume la responsabilidad de articular propuestas curriculares dirigidas a la deconstrucción del estereotipo y la superación de representaciones sociales excluyentes.

La destacada presencia de alumnado latinoamericano en las aulas españolas impulsa la urgente delimitación teórica y procedimental de la dimensión iberoamericana, tal y como ya puede comprobarse en la consolidación de la idea de Europa o la definición del espacio e identidad europea en las normativas curriculares y textos escolares para la Educación Primaria en España.

\section{Presencias y ausencias} curriculares para la consolidación del Espacio Cultural Iberoamericano

\section{Metodología}

Para examinar los contenidos, criterios de evaluación y estándares de aprendizaje evaluables de las concreciones curriculares autonómicas de la $\mathrm{LOMCE}^{1}$,

\footnotetext{
${ }^{1}$ Para la conformación de la muestra objeto de análisis, han sido consideradas las normativas curriculares publicadas hasta el 31 de agosto de 2015, momento de finalización de la presente investigación.
} 
seguimos una metodología de análisis de contenido descriptivo e inferencial, considerando tanto los contenidos manifiestos como los latentes (López-Aranguren, 1990). La interpretación cualitativa de los contenidos ha sido realizada sobre el registro cuantitativo del contenido manifiesto, clasificando las unidades de registro (palabras clave) -cuyas características han determinado su adscripción categórica- en la unidad de contexto que la explica y concreta (Krippendoff, 1990; Gil, 1994). Según esto, en el vaciado de las palabras clave consideradas (América, Iberoamérica, diversidad cultural e interculturalidad), hemos priorizado las unidades contextuales en las que se produce su aparición. La definición previa de la categoría de análisis orienta la investigación a posicionamientos deductivos de los contenidos, criterios de evaluación y estándares de aprendizaje evaluables objeto de estudio (Tabla I).

De acuerdo a estos principios metodológicos, recogemos los contenidos, criterios y estándares referidos América e Iberoamérica de forma expresa. En el vaciado de datos no consideramos, sin embargo, los ejes temáticos globalizadores en torno a los descubrimientos, a

Tabla I. Enfoques curriculares.

\begin{tabular}{|c|l|l|}
\hline Categoría & \multicolumn{1}{|c|}{ Indicador } & \multicolumn{1}{c|}{ Descriptor } \\
\hline & $\begin{array}{l}\text { Segmentario/ } \\
\text { parcial }\end{array}$ & $\begin{array}{l}\text { Los contenidos de interés iberoamericano aparecen } \\
\text { vinculados al tratamiento curricular de una etapa } \\
\text { determinada de la Historia, excluyendo su presencia } \\
\text { en otras de significativa relevancia en la explicación } \\
\text { de procesos histórico-culturales. }\end{array}$ \\
\cline { 2 - 4 } Enfoque & $\begin{array}{l}\text { Historicista/ } \\
\text { enciclopédico }\end{array}$ & $\begin{array}{l}\text { Los contenidos de interés iberoamericano aparecen } \\
\text { vinculados al tratamiento curricular de las etapas his- } \\
\text { tóricas con un carácter compendioso/compilatorio de } \\
\text { contenidos. }\end{array}$ \\
\cline { 2 - 3 } & $\begin{array}{l}\text { Procesual/ } \\
\text { explicativo }\end{array}$ & $\begin{array}{l}\text { Los contenidos de interés iberoamericano aparecen } \\
\text { vinculados al tratamiento curricular de procesos his- } \\
\text { tóricos, priorizando su naturaleza procesual y causal } \\
\text { en la configuración de paradigmas culturales actuales. }\end{array}$ \\
\cline { 2 - 3 } & $\begin{array}{l}\text { Los contenidos de interés iberoamericano aparecen } \\
\text { vinculados al tratamiento curricular de procesos his- } \\
\text { tóricos, priorizando su naturaleza procesual y causal } \\
\text { de paradigmas culturales actuales, contribuyendo a } \\
\text { su proyección y desarrollo de futuro. }\end{array}$ \\
\hline proyectista
\end{tabular}

Fuente: Elaboración propia. 
la idea imperial de la monarquía hispánica y a los viajes de expansión maríti$m a$, por no aparecer concretados en su formulación contenidos americanistas específicos, dirigidos a sostener y argumentar una dimensión iberoamericana precisa.

Finalmente, hemos centrado nuestra atención en el Bloque 4. Las huellas en el tiempo, correspondiente al quinto y sexto curso de Educación Primaria, pues aborda, de forma sistemática, los contenidos históricos objeto de estudio.

Análisis y discusión de resultados.

El Real Decreto 1513/2006, a través de su concepción competencial, ya proponía el tratamiento de la competencia social y ciudadana como núcleo para la comprensión de la pluralidad de las sociedades actuales y de la contribución de las diferentes culturas al desarrollo de la humanidad, orientadas ambas a la educación para una ciudadanía global diversa, compleja y múltiple. Sin embargo, los exiguos contenidos americanistas no eran vinculados a su potencial naturaleza intercultural y a su inserción en procesos históricos y culturales compartidos que, en última instancia, frenaban su presencia en su relación con las políticas monárquicas peninsulares de los siglos XV, XVI, XVII y XVIII (Ortega, 2015).

El vigente Real Decreto 126/2014, mediante la inclusión expresa de las competencias sociales y civicas $\mathrm{y}$, de forma específica, de la justificación del área de valores sociales y cívicos, des- taca la necesidad de comprender la dimensión intercultural y de percibir el carácter dinámico, cambiante e interactivo de las identidades culturales en el contexto nacional, europeo y global. Asimismo, apunta la comprensión de los procesos migratorios y su determinación en la configuración de las sociedades multiculturales en un mundo globalizado. Igualmente, justifica el área de Ciencias Sociales en la valoración de la diversidad, en todos sus ámbitos, como elemento de enriquecimiento cultural. Además, en el aprendizaje de las lenguas extranjeras recuerda "uno de los principios inspiradores de la Unión: la unidad en la diversidad" (Real Decreto $126 / 2014$, p. 19410), precisamente el atribuido a la preexistencia de un Espacio Cultural Iberoamericano, en este caso, absolutamente ausente.

Los primeros resultados (Anexo I) informan de una clara recuperación del tradicional discurso nacional y su encaje geopolítico europeo, alejando, más si cabe, los referentes culturales iberoamericanos. En efecto, desde una perspectiva eminentemente política, estos referentes se restringen al descubrimiento de América como hito histórico universal e inicio de la Edad Moderna, a las causas que motivaron las expediciones marítimas, a la monarquía de los Reyes Católicos, a las repercusiones del descubrimiento, y a la expansión y administración imperial de los territorios de la monarquía hispánica bajo los reinados de Carlos I y Felipe II, en la que el término América desaparece. Las disposiciones curriculares, que inclu- 
yen el continente americano como contenido específico de procesos históricos, aluden el referente continental americano para abordar el inicio de la Edad Moderna y el reinado de los Reyes Católicos, sin mayor lectura ni tratamiento intercultural. En algún caso, este tratamiento podría estar presumiblemente integrado en imprecisos estándares de aprendizaje como "las repercusiones del descubrimiento de América" (Orden Edu/519/2014 -Castilla y León-). En ocasiones, estos contenidos derivan en especificaciones de cierto carácter enciclopedista, recogiendo, de forma concreta, los viajes de Cristóbal Colón, y la identificación de algunos conquistadores, navegantes y exploradores como Hernán Cortés, Pizarro, Magallanes y Elcano (Decreto 89/2014 -Madrid-); o la ruta del primer viaje de Colón y la explicación del almirante de la adjudicación del nombre de "indios" a los nativos americanos (Decreto 54/2014 -Castilla-La Mancha-).

En 8 regulaciones, ni siquiera aparece el nombre de América -más allá de su conexión con el estudio lingüístico del español- en relación al tratamiento curricular de las Ciencias Sociales (Decreto 105/2014 -Galicia-; Decreto 198/2014 -Murcia-; Decreto 32/2014 Illes Balears-; Decreto 103/2014 -Extremadura-; Decret 119/2015 -Catalunya-; Decreto 89/2014 -Canarias-; Decreto 82/2014 -Principado de Asturias-; Orden de 16 de junio de 2014 -Aragón-). La presencia del continente americano aparece supeditada, sin embargo, a tres grandes ejes temáticos como "La época de los descubrimientos", "Las grandes expediciones marítimas" o, más frecuentemente, "La monarquía hispánica en la Edad Moderna". Los contenidos americanistas e iberoamericanos específicos no encuentran continuidad al concepto subyacente de imperio, irremisiblemente unido al de América en estas disposiciones curriculares.

Aunque de marcado carácter nacionalista y europeísta, de las 17 concreciones curriculares autonómicas publicadas hasta la fecha para la etapa de Educación Primaria, sólo las regulaciones de la Comunidad de Madrid y de la Comunidad de Castilla-La Macha contemplan contenidos, criterios de evaluación y estándares de aprendizaje relacionados con los procesos independentistas, mediante la identificación del primer cuarto del siglo XIX como el de la emancipación de la América continental, y el año 1898 como el de la pérdida de las últimas colonias (Decreto 89/2014-Madrid-); la pérdida de las colonias en América y el Pacífico, y la emancipación de las colonias durante el reinado de Fernando VII (Decreto 54/2014 -Castilla-La Mancha).

Contrariamente, en el currículo de la Comunidad Valenciana (Decreto $108 / 2014$ ), resulta muy interesante la incorporación de contenidos, en clave comparativa, relacionados con procesos migratorios históricos en su Bloque 3 . Vivir en sociedad para el área de Ciencias Sociales de quinto curso. Entre estos procesos, muy atinadamente se incluye la colonización de América, la expansión hacia América y las migra- 
ciones europeas durante el siglo XIX, concretadas en su criterio de evaluación BL3.3 "Comparar diversos fenómenos migratorios con los actuales relativos a España y la Unión Europea desde el punto de vista de las causas y la región de su origen y la de su destino como la emigración española y europea a América o la expulsión de los judíos y moriscos de los territorios de la Monarquía Hispánica" (p. 16531). La particularidad de estos contenidos, fundamentales en la comprensión de la complejidad de las sociedades multiculturales actuales, no aparece, sin embargo, en ninguna otra regulación.

Finalmente, son invisibles los contenidos clave vinculados a las culturas precolombinas y a los lazos de unión comunitarios transnacionales, fortalecidos, anualmente, en las Cumbres Iberoamericanas de Jefes de Estado y Presidentes de Gobierno. El tratamiento de la interculturalidad y la diversidad cultural en el espacio iberoamericano, marco de desarrollo geo-cultural de Iberoamérica -término también ausente en las disposiciones-, es inexistente. Como ya sucedía en el Real Decreto 1513/2006 y en sus regulaciones autonómicas, los contenidos referentes a América y/o a Iberoamérica se presentan completamente desvinculados de su potencial tratamiento intercultural en las nuevas normativas. Esta desconexión constituye la pérdida de una excelente oportunidad para el trabajo de la diversidad cultural y la multiculturalidad en el aula, a través de la selección de contenidos en los que el alumnado iberoamericano en
España pudiera encontrar referentes válidos, trascendiendo planteamientos eurocéntricos que en poco, o en nada, remiten a la complejidad histórica de procesos compartidos en la región.

Resulta reveladora la frecuencia terminológica general en la conformación de otras dimensiones supranacionales como la europea en el currículo básico de la LOMCE para Educación Primaria: 2 apariciones, para lo que podría delimitar una cierta realidad iberoamericana, frente a las 43 menciones terminológicas sobre Europa. Esta presencia resulta significativa, y recurrente en los currícula autonómicos, en el estándar de aprendizaje evaluable del bloque 5.2 "Aprecia la herencia cultural a escala local, nacional y europea como riqueza compartida que hay que conocer, preservar y cuidar" (Real Decreto 126/2014, p. 19377). Si diversificamos las palabras clave América y Europa en otras derivadas como ciudadanía europea / iberoamericana e identidad cultural europea / iberoamericana -incluyendo en ellas las referidas a las características lingüísticas del español de América-, estas frecuencias son igualmente proporcionales en las regulaciones autonómicas (Anexo II).

De acuerdo a los resultados, la potencial dimensión iberoamericana desaparece para impulsar una clara dimensión europea, con la insistencia curricular en el conocimiento y valoración del patrimonio local, nacional y europeo como riqueza compartida, el conocimiento de las instituciones europeas y el lugar de España en el conjunto de la Unión. En 
este sentido, confirmamos la adopción curricular de enfoques segmentarios/ parcialistas y, anecdóticamente, historicistas/enciclopédicos (Decreto 89/2014 -Madrid-; Decreto 54/2014 -CastillaLa Mancha-) y procesuales/explicativos (Decreto 108/2014 -Comunitat Valenciana-). Resultan ausentes, en cambio, los enfoques procesuales/proyectistas.

La valoración de la herencia cultural a escala iberoamericana permanece invisible en el relato nacional y europeísta de la nueva ley de educación; un aspecto fácilmente abordable, cuando menos, desde el tratamiento de su patrimonio histórico-cultural. En esta dirección, son relevantes los avances realizados por el Ministerio de Educación, Cultura y Deporte de España en materia de patrimonio documental iberoamericano, con el acceso digital a un ya importante volumen de documentos de interés iberoamericanista en el Portal del Bicentenario de las Independencias Iberoamericanas, el Portal de Movimimientos Migratorios Iberoamericanos (http://pares.mcu.es/) y en el Censo-Guía de Archivos de España e Iberoamérica (http://censoarchivos. mcu.es/CensoGuia/portada.htm).

\section{Conclusiones}

En la línea de las conclusiones del reciente estudio comparativo de Bellatti, Gámez y Trepat (2015) para el caso de la Educación Secundaria española, portuguesa y de cuatro países del ámbito latinoamericano (México, Ecuador, Argentina y Brasil), el tratamiento curricular de Iberoamérica en la Educación Primaria en España se dirige hacia la fundamentación/argumentación de un marcado relato nacional, sostén de ubicación espacio-temporal que imposibilita una posible definición de realidades supranacionales, excepción hecha a la europea.

A la espera del análisis de los resultados acerca de las representaciones sociales de la Historia de Iberoamérica en el alumnado de sexto curso de Educación Primaria, contamos con los resultados de un reciente estudio realizado a 1961 alumnos procedentes de distintas nacionalidades matriculados en distintos centros educativos españoles de Educación Secundaria Obligatoria (Molina, Fuentes y Alfageme, 2015; Prats, Valls y Miralles, 2015); concluye, de acuerdo al vaciado de datos, la ausencia de contenidos propios de la historia común iberoamericana en los conocimientos consolidados al finalizar la Educación Secundaria Obligatoria, al tiempo que observa confusión en los estudiantes en la determinación de los países que integran la Comunidad Iberoamericana de Nacionales, y una notable imprecisión en aspectos vinculados a la historia iberoamericana.

Las concreciones conceptuales de la Historia en la LOMCE obstaculiza la construcción de la idea de Iberoamérica y del Espacio Cultural Iberoamericano, reconocido y consolidado desde la declaración de los principios y fines de la Carta Cultural Iberoamericana. Este espacio no parece corresponder con las normativas autonómicas ni con los 
conocimientos con los que el alumnado finalizará, tras la superación de los estudios primarios, su etapa de escolarización obligatoria; una cuestión preocupante y urgente que ya ha llevado a algunos didactas de la historia a negar, para el caso español, la existencia de una verdadera dimensión iberoamericana en la enseñanza y en el aprendizaje de la Historia.

Coincidimos con Amblés (2009) en fijar la atención, desde un punto de vista metodológico, en los beneficios del trabajo conjunto en torno a una historia, por más de tres siglos compartida, para "dar respuestas y favorecer la diversidad cultural al mismo tiempo que el tratamiento adecuado de la interculturalidad en nuestras aulas" (p. 67).

\section{Referencias bibliográficas}

AMBLÉS, S. (2009). España en América y América en España: un reto escolar. Tarbiya: Revista de investigación e innovación educativa, $\mathrm{n}^{\circ}$ 40, 63-80.

BELLATTI, B., GÁMEZ, V. y TREPAT, C. (2015). Los contenidos curriculares de historia en los países del ámbito iberoamericano: una aproximación cuantitativa. En J. PRATS, R. VALLS y P. MIRALLES (eds.), Iberoamérica en las aulas. Qué estudia y qué sabe el alumnado de Educación Secundaria (21-102). Lleida: Editorial Milenio.

GIL FLORES, J. (1994). Análisis de datos cualitativos. Aplicaciones a la investigación educativa. Barcelona: PPU.
KRIPPENDOFF, K. (1990). Metodología del análisis de contenido. Teoría y práctica. Barcelona: Paidós.

LÓPEZ ARANGUREN, E. (1990). El análisis de contenido. En M. GARCÍA FERRANDO, J. IBÁÑEZ y F. ALVIRA (comp.), El análisis de la realidad social (383-414). Madrid: Alianza.

MOLINA, S.; FUENTES, C; ALFAGEME, M ${ }^{a}$ B. (2015). ¿Qué saben de la historia iberoamericana los estudiantes de $4^{\circ}$ de la Educación Secundaria Obligatoria? En J. PRATS, R. VALLS y P. MIRALLES (eds.), Iberoamérica en las aulas. Qué estudia y qué sabe el alumnado de Educación Secundaria (233-292). Lleida: Editorial Milenio.

ORTEGA SÁNCHEZ, D. (2015). Didáctica de la Historia y construcción de la identidad cultural iberoamericana en el currículo y libros de texto de Ciencias Sociales de Educación Primaria españoles. Burgos: Universidad de Burgos (Tesis Doctoral inédita, dirigida por J. A. Jiménez Eguizábal y F. Rodríguez Lestegás).

PRATS, J ; MIRALLES, P. y VALLS FIGUERA, R. G. (2015). Quiénes saben más y quiénes menos: las diferencias de conocimientos entre el alumnado. En J. PRATS, R. VALLS y P. MIRALLES (eds.), Iberoamérica en las aulas. Qué estudia y qué sabe el alumnado de Educación Secundaria (293-312). Lleida: Editorial Milenio.

VALLS, R. (2012). La enseñanza española de la historia y su dimensión Iberoamericana. Didáctica de las Cien- 
cias Experimentales y Sociales, $\mathrm{n}^{\circ} 26$, 121-143. DOI: 10.7203/DCES.26.1936

VALLS, R. (2011). La multiculturalidad en la enseñanza de la Historia y el desajuste entre intenciones educativas y prácticas escolares: los retos del presente y del futuro inmediato. Educar em Revista, Curitiba, Brasil, $\mathrm{n}^{\circ} 42$, 73-94. DOI: $10.1590 / \mathrm{S} 0104-$ 40602011000500006

\section{Normativas}

Carta Cultural Iberoamericana (2006).

Montevideo-Uruguay. Accesible en: http://www.culturasiberoamericanas. org/carta_cultural.php [15 de julio de 2015].

Real Decreto 126/2014, de 28 de febrero, por el que se establece el currículo básico de la Educación Primaria. Boletín Oficial del Estado, $\mathrm{n}^{\circ} 52$.

Real Decreto 1513/2006, de 7 de diciembre, por el que se establecen las enseñanzas mínimas de la Educación Primaria. Boletín Oficial del Estado, ${ }^{\circ}$ 293. 


\section{Anexo I.}

Contenidos, criterios de evaluación y estándares de aprendizaje evaluables de las concreciones curriculares autonómicas de la LOMCE.

Fuente: Elaboración propia.

«Orden de 17 de marzo de 2015, por la que se desarrolla el currículo correspondiente a la Educación Primaria en Andalucía». Boletín Oficial de la Junta de Andalucía, núm. 60.

\begin{tabular}{|c|l|l|c|c|}
\hline $\begin{array}{c}\text { Curso/ } \\
\text { Ciclo }\end{array}$ & Bloque & Contenidos especificos & $\begin{array}{l}\text { Criterios de } \\
\text { evaluación }\end{array}$ & $\begin{array}{c}\text { Estándares de } \\
\text { aprendizaje }\end{array}$ \\
\hline 3 Ciclo & $\begin{array}{l}\text { 4. Las } \\
\text { huellas en } \\
\text { el tiempo. }\end{array}$ & $\begin{array}{l}\text { 4.2. El Reinado de los } \\
\text { Reyes Católicos: la } \\
\text { unión dinástica, la } \\
\text { conquista de Granada, la } \\
\text { expulsión de los judíos, } \\
\text { el descubrimiento de } \\
\text { América. }\end{array}$ & - & - \\
\hline
\end{tabular}

«Orden de 16 de junio de 2014, de la Consejera de Educación, Universidad, Cultura y Deporte, por la que se aprueba el currículo de la Educación Primaria y se autoriza su aplicación en los centros docentes de la Comunidad Autónoma de Aragón». Boletín Oficial de Aragón, núm. 119.

\begin{tabular}{|c|c|c|c|c|}
\hline $\begin{array}{c}\text { Cursol } \\
\text { Ciclo }\end{array}$ & Bloque & Contenidos específicos & $\begin{array}{c}\text { Criterios de } \\
\text { evaluación }\end{array}$ & $\begin{array}{c}\text { Estándares de } \\
\text { aprendizaje }\end{array}$ \\
\hline- & - & - & - & - \\
\hline
\end{tabular}

«Decreto 82/2014, de 28 de agosto, por el que se regula la ordenación y establece el currículo de la Educación Primaria en el Principado de Asturias». Boletín Oficial del Principado de Asturias, núm. 202.

\begin{tabular}{|c|c|c|c|c|}
\hline $\begin{array}{c}\text { Cursol } \\
\text { Ciclo }\end{array}$ & Bloque & Contenidos específicos & $\begin{array}{c}\text { Criterios de } \\
\text { evaluación }\end{array}$ & $\begin{array}{c}\text { Estándares de } \\
\text { aprendizaje }\end{array}$ \\
\hline- & - & - & - & - \\
\hline
\end{tabular}


«Decreto 108/2014, de 4 de julio, del Consell, por el que establece el currículo y desarrolla la ordenación general de la educación primaria en la Comunitat Valenciana». Diari Oficial de la Comunitat Valenciana, núm.

\begin{tabular}{|c|c|l|l|l|}
\hline $\begin{array}{c}\text { Curso/ } \\
\text { Ciclo }\end{array}$ & Bloque & Contenidos específicos & \multicolumn{1}{|c|}{$\begin{array}{c}\text { Criterios de } \\
\text { evaluación }\end{array}$} & $\begin{array}{c}\text { Estándares de } \\
\text { aprendizaje }\end{array}$ \\
\hline $5^{\circ}$ & $\begin{array}{l}3 . \text { Vivir en } \\
\text { sociedad }\end{array}$ & $\begin{array}{l}\text { Movimientos migratorios } \\
\text { históricos: expulsiones } \\
\text { de judíos y moriscos, } \\
\text { colonización de América } \\
\text { migraciones europeas del } \\
\text { siglo XIX. }\end{array}$ & $\begin{array}{l}\text { lL3.3. Comparar } \\
\text { diversos fenómenos } \\
\text { migratorios históricos } \\
\text { con los actuales } \\
\text { relativos a España y la } \\
\text { Unión Europea desde } \\
\text { el punto de vista de } \\
\text { las causas y la región } \\
\text { de su origen y la de } \\
\text { su destino como la } \\
\text { emigración española } \\
\text { y europea a América } \\
\text { o la expulsión de los } \\
\text { judíos y moriscos de } \\
\text { los territorios de la } \\
\text { Monarquía Hispánica. }\end{array}$ & \\
\hline $5^{\circ}$ & $\begin{array}{l}\text { 4. Las } \\
\text { huellas } \\
\text { del } \\
\text { tiempo. }\end{array}$ & $\begin{array}{l}\text { La formación de la } \\
\text { Monarquía Hispánica y su } \\
\text { expansión en América y } \\
\text { Europa. }\end{array}$ & \\
\hline
\end{tabular}

«Decreto 89/2014, de 1 de agosto, por el que se establece la ordenación y el currículo de la Educación Primaria en la Comunidad Autónoma de Canarias». Boletín Oficial de Canarias, núm. 156.

\begin{tabular}{|c|c|c|c|c|}
\hline $\begin{array}{c}\text { Cursol } \\
\text { Ciclo }\end{array}$ & Bloque & Contenidos específicos & $\begin{array}{c}\text { Criterios de } \\
\text { evaluación }\end{array}$ & $\begin{array}{c}\text { Estándares de } \\
\text { aprendizaje }\end{array}$ \\
\hline- & - & - & - & - \\
\hline
\end{tabular}




\begin{tabular}{|c|c|c|c|c|}
\hline \multicolumn{5}{|c|}{$\begin{array}{c}\text { «ecreto 27/2014, de } 5 \text { de junio, que establece el currículo de Educación } \\
\text { Primaria en la Comunidad Autónoma de Cantabria». Boletín Oficial de } \\
\text { Cantabria, núm. } 29 .\end{array}$} \\
\hline $\begin{array}{l}\text { Cursol } \\
\text { Ciclo }\end{array}$ & Bloque & Contenidos específicos & $\begin{array}{l}\text { Criterios de } \\
\text { evaluación }\end{array}$ & $\begin{array}{c}\text { Estándares de } \\
\text { aprendizaje }\end{array}$ \\
\hline $5^{\circ}$ & $\begin{array}{l}\text { 4. Las } \\
\text { huellas } \\
\text { del } \\
\text { tiempo. }\end{array}$ & $\begin{array}{l}\text { El Reinado de los Reyes } \\
\text { Católicos: la unión dinástica, } \\
\text { la conquista de Granada, } \\
\text { la expulsión de los judíos, } \\
\text { el descubrimiento de } \\
\text { América. }\end{array}$ & - & - \\
\hline
\end{tabular}

\begin{tabular}{|c|l|l|l|l|}
\hline \multicolumn{3}{|c|}{ «Decreto 54/2014, de 10/07/2014, por el que se establece el currículo de } \\
la Educación Primaria en la Comunidad Autónoma de Castilla-La \\
Mancha». Diario Oficial de Castilla-La Mancha, \\
núm. 132.
\end{tabular}


«Decret 119/2015, de 23 de juny, d'ordenació dels ensenyaments de l'educació primària». Diari Oficial de la Generalitat de Catalunya, núm. 6900.

\begin{tabular}{|c|c|c|c|c|}
\hline $\begin{array}{c}\text { Cursol } \\
\text { Ciclo }\end{array}$ & Bloque & Contenidos específicos & $\begin{array}{c}\text { Criterios de } \\
\text { evaluación }\end{array}$ & $\begin{array}{c}\text { Estándares de } \\
\text { aprendizaje }\end{array}$ \\
\hline- & - & - & - & - \\
\hline
\end{tabular}

«ORDEN EDU/519/2014, de 17 de junio, por la que se establece el currículo y se regula la implantación, evaluación y desarrollo de la educación primaria en la Comunidad de Castilla y León». Boletín Oficial de Castilla y León, núm. 117.

\begin{tabular}{|c|l|l|l|l|}
\hline $\begin{array}{c}\text { Cursol } \\
\text { Ciclo }\end{array}$ & Bloque & Contenidos específicos & $\begin{array}{l}\text { Criterios de } \\
\text { evaluación }\end{array}$ & $\begin{array}{c}\text { Estándares de } \\
\text { aprendizaje }\end{array}$ \\
\hline $5^{\circ}$ & $\begin{array}{l}\text { 4. Las } \\
\text { huellas } \\
\text { del } \\
\text { tiempo. }\end{array}$ & $\begin{array}{l}\text { El reinado de los Reyes } \\
\text { Católicos: la unión dinástica, } \\
\text { la conquista de Granada, } \\
\text { la expulsión de los judíos, } \\
\text { el descubrimiento de } \\
\text { América. }\end{array}$ & - & $\begin{array}{l}\text { 3.3. Analiza las } \\
\text { repercusiones del } \\
\text { descubrimiento de } \\
\text { América. }\end{array}$ \\
\hline
\end{tabular}

«Decreto 103/2014, de 10 de junio, por el que se establece el currículo de Educación Primaria para la Comunidad Autónoma de Extremadura». Diario Oficial de Extremadura, núm. 114.

\begin{tabular}{|c|c|c|c|c|}
\hline $\begin{array}{c}\text { Cursol } \\
\text { Ciclo }\end{array}$ & Bloque & Contenidos especificos & $\begin{array}{c}\text { Criterios de } \\
\text { evaluación }\end{array}$ & $\begin{array}{c}\text { Estándares de } \\
\text { aprendizaje }\end{array}$ \\
\hline- & - & - & - & - \\
\hline
\end{tabular}

«Decreto 105/2014, do 4 de setembro, polo que se establece o currículo da educación primaria na Comunidade Autónoma de Galicia». Diario Oficial de Galicia, núm. 171.

\begin{tabular}{|c|c|c|c|c|}
\hline $\begin{array}{c}\text { Cursol } \\
\text { Ciclo }\end{array}$ & Bloque & Contenidos específicos & $\begin{array}{c}\text { Criterios de } \\
\text { evaluación }\end{array}$ & $\begin{array}{c}\text { Estándares de } \\
\text { aprendizaje }\end{array}$ \\
\hline- & - & - & - & - \\
\hline
\end{tabular}

«Decreto 32/2014 de 18 de julio, por el que se establece el currículo de la educación primaria en las Illes Balears». Butlletí Oficial de les Illes Balears, núm. 97.

\begin{tabular}{|c|c|c|c|c|}
\hline $\begin{array}{c}\text { Cursol } \\
\text { Ciclo }\end{array}$ & Bloque & Contenidos especificos & $\begin{array}{c}\text { Criterios de } \\
\text { evaluación }\end{array}$ & $\begin{array}{c}\text { Estándares de } \\
\text { aprendizaje }\end{array}$ \\
\hline- & - & - & - & - \\
\hline
\end{tabular}




\begin{tabular}{|c|c|c|c|c|}
\hline \multicolumn{5}{|c|}{$\begin{array}{c}\text { «ecreto 24/2014, de } 13 \text { de junio, por el que se establece el currículo de la } \\
\text { Educación Primaria en la Comunidad Autónoma de La Rioja». Boletín } \\
\text { Oficial de La Rioja, núm. } 74 .\end{array}$} \\
\hline $\begin{array}{l}\text { Cursol } \\
\text { Ciclo }\end{array}$ & Bloque & Contenidos específicos & $\begin{array}{l}\text { Criterios de } \\
\text { evaluación }\end{array}$ & $\begin{array}{c}\text { Estándares de } \\
\text { aprendizaje }\end{array}$ \\
\hline $5^{\circ}$ & $\begin{array}{l}\text { 4. Las } \\
\text { huellas } \\
\text { del } \\
\text { tiempo. }\end{array}$ & $\begin{array}{l}\text { El Reinado de los } \\
\text { Reyes Católicos: la } \\
\text { unión dinástica, la } \\
\text { conquista de Granada, la } \\
\text { expulsión de los judíos, } \\
\text { el descubrimiento de } \\
\text { América. }\end{array}$ & - & - \\
\hline
\end{tabular}

\begin{tabular}{|c|c|c|c|c|}
\hline \multicolumn{5}{|c|}{$\begin{array}{c}\text { «Decreto 89/2014, de } 24 \text { de julio, del Consejo de Gobierno, por el que se } \\
\text { establece para la Comunidad de Madrid el Currículo de la Educación } \\
\text { Primaria». Boletín Oficial de la Comunidad de Madrid, núm. } 175 .\end{array}$} \\
\hline $\begin{array}{l}\text { Cursol } \\
\text { Ciclo }\end{array}$ & Bloque & Contenidos específicos & $\begin{array}{l}\text { Criterios de } \\
\text { evaluación }\end{array}$ & $\begin{array}{c}\text { Estándares de } \\
\text { aprendizaje }\end{array}$ \\
\hline $4^{\circ}$ & $\begin{array}{l}\text { 4. La } \\
\text { huella del } \\
\text { tiempo. }\end{array}$ & - & - & $\begin{array}{l}\text { 25. Identifica el } \\
\text { descubrimiento de } \\
\text { América (1492) como } \\
\text { la entrada de España } \\
\text { en la Edad Moderna. }\end{array}$ \\
\hline $5^{\circ}$ & $\begin{array}{l}\text { 4. La } \\
\text { huella del } \\
\text { tiempo. }\end{array}$ & $\begin{array}{l}\text { Los Reyes Católicos. } \\
\text { El descubrimiento de } \\
\text { América. }\end{array}$ & - & $\begin{array}{l}\text { 19. Identifica a algu- } \\
\text { nos conquistadores, } \\
\text { navegantes y explo- } \\
\text { radores como Her- } \\
\text { nán Cortés, Pizarro, } \\
\text { Magallanes o Elcano. } \\
\text { 21. Explica el descu- } \\
\text { brimiento de Améri- } \\
\text { ca (1492) y los viajes } \\
\text { de Cristóbal Colón. }\end{array}$ \\
\hline $6^{\circ}$ & $\begin{array}{l}\text { 4. La } \\
\text { huella del } \\
\text { tiempo. }\end{array}$ & - & - & $\begin{array}{l}\text { 13. Identifica el primer } \\
\text { cuarto del siglo XIX } \\
\text { como el de la indepen- } \\
\text { dencia de la América } \\
\text { continental. } \\
\text { 16. Identifica } 1898 \\
\text { como el año de la pér- } \\
\text { dida de las últimas } \\
\text { posesiones de ultra- } \\
\text { mar: Cuba, Puerto } \\
\text { Rico y Filipinas. }\end{array}$ \\
\hline
\end{tabular}


«Orden ECD/686/2014, de 23 de abril, por la que se establece el currículo de la Educación Primaria para el ámbito de gestión del Ministerio de Educación, Cultura y deporte y se regula su implantación, así como la evaluación y determinados aspectos organizativos de la etapa». Boletín Oficial del Estado, núm. 106.

\begin{tabular}{|c|l|l|c|c|}
\hline $\begin{array}{c}\text { Cursol } \\
\text { Ciclo }\end{array}$ & Bloque & Contenidos especificos & $\begin{array}{l}\text { Criterios de } \\
\text { evaluación }\end{array}$ & $\begin{array}{c}\text { Estándares de } \\
\text { aprendizaje }\end{array}$ \\
\hline $5^{\text {o }}$ & $\begin{array}{l}\text { 4. Las } \\
\text { huellas } \\
\text { del } \\
\text { tiempo. }\end{array}$ & $\begin{array}{l}\text { El Reinado de los } \\
\text { Reyes Católicos: la } \\
\text { unión dinástica, la } \\
\text { conquista de Granada, la } \\
\text { expulsión de los judíos, } \\
\text { el descubrimiento de } \\
\text { América. }\end{array}$ & - & - \\
\hline
\end{tabular}

«Decreto 198/2014, de 5 de septiembre, por el que se establece el currículo de la Educación Primaria en la Comunidad Autónoma de la Región de Murcia». Boletín Oficial de la Región de Murcia, núm. 206.

\begin{tabular}{|c|c|c|c|c|}
\hline $\begin{array}{c}\text { Cursol } \\
\text { Ciclo }\end{array}$ & Bloque & Contenidos específicos & $\begin{array}{c}\text { Criterios de } \\
\text { evaluación }\end{array}$ & $\begin{array}{c}\text { Estándares de } \\
\text { aprendizaje }\end{array}$ \\
\hline- & - & - & - & - \\
\hline
\end{tabular}

«Decreto Foral 60/2014, de 16 de julio, por el que se establece el currículo de las enseñanzas de Educación primaria en la Comunidad Foral de

Navarra». Boletín Oficial de Navarra, núm. 174.

\begin{tabular}{|c|l|l|c|c|}
\hline $\begin{array}{c}\text { Cursol } \\
\text { Ciclo }\end{array}$ & Bloque & Contenidos específicos & $\begin{array}{l}\text { Criterios de } \\
\text { evaluación }\end{array}$ & $\begin{array}{c}\text { Estándares de } \\
\text { aprendizaje }\end{array}$ \\
\hline $5^{\circ}$ & $\begin{array}{l}\text { 4. Las } \\
\text { huellas } \\
\text { del } \\
\text { tiempo. }\end{array}$ & $\begin{array}{l}\text { El Reinado de los } \\
\text { Reyes Católicos: la } \\
\text { unión dinástica, la } \\
\text { conquista de Granada, la } \\
\text { expulsión de los judíos, } \\
\text { el descubrimiento de } \\
\text { América. }\end{array}$ & - & - \\
\hline
\end{tabular}




\section{Anexo II. Frecuencias conceptuales y terminológicas.}

Fuente: Elaboración propia.

«Orden de 17 de marzo de 2015, por la que se desarrolla el currículo correspondiente a la Educación Primaria en Andalucía». Boletín Oficial de la Junta de Andalucía, núm. 60.

\begin{tabular}{|l|c|l|c|}
\hline \multicolumn{2}{|c|}{ Ámbito europeo } & \multicolumn{2}{c|}{ Ámbito iberoamericano } \\
\hline Palabra clave & Frecuencia & Palabra clave & Frecuencia \\
\hline Europa & 42 & América & 3 \\
\hline Unión Europea & 41 & Iberoamérica & - \\
\hline Ciudadanía europea & - & Ciudadanía iberoamericana & - \\
\hline Identidad europea & - & Identidad iberoamericana & - \\
\hline
\end{tabular}

«Orden de 16 de junio de 2014, de la Consejera de Educación, Universidad, Cultura y Deporte, por la que se aprueba el currículo de la Educación Primaria y se autoriza su aplicación en los centros docentes de la Comunidad Autónoma de Aragón». Boletín Oficial de Aragón, núm. 119.

\begin{tabular}{|l|c|l|c|}
\hline \multicolumn{2}{|c|}{ Ámbito europeo } & \multicolumn{2}{c|}{ Ámbito iberoamericano } \\
\hline Palabra clave & Frecuencia & Palabra clave & Frecuencia \\
\hline Europa & 37 & América & 2 \\
\hline Unión Europea & 47 & Iberoamérica & - \\
\hline Ciudadanía europea & 1 & Ciudadanía iberoamericana & - \\
\hline Identidad europea & 1 & Identidad iberoamericana & - \\
\hline
\end{tabular}

«Decreto 82/2014, de 28 de agosto, por el que se regula la ordenación y establece el currículo de la Educación Primaria en el Principado de Asturias». Boletín Oficial del Principado de Asturias, núm. 202.

\begin{tabular}{|l|c|l|c|}
\hline \multicolumn{2}{|c|}{ Ámbito europeo } & \multicolumn{2}{c|}{ Ámbito iberoamericano } \\
\hline Palabra clave & Frecuencia & Palabra clave & Frecuencia \\
\hline Europa & 24 & América & 8 \\
\hline Unión Europea & 22 & Iberoamérica & - \\
\hline Ciudadanía europea & - & Ciudadanía iberoamericana & - \\
\hline Identidad europea & - & Identidad iberoamericana & - \\
\hline
\end{tabular}




\begin{tabular}{|c|c|c|c|}
\hline \multicolumn{4}{|c|}{$\begin{array}{c}\text { «ecreto 108/2014, de } 4 \text { de julio, del Consell, por el que establece el } \\
\text { currículo y desarrolla la ordenación general de la educación primaria } \\
\text { en la Comunitat Valenciana». Diari Oficial de la Comunitat Valenciana, } \\
\text { núm. } 7311 .\end{array}$} \\
\hline \multicolumn{2}{|c|}{ Ámbito europeo } & \multicolumn{2}{|c|}{ Ámbito iberoamericano } \\
\hline Palabra clave & Frecuencia & Palabra clave & Frecuencia \\
\hline Europa & 11 & América & 7 \\
\hline Unión Europea & 5 & Iberoamérica & - \\
\hline Ciudadanía europea & 3 & Ciudadanía iberoamericana & - \\
\hline Identidad europea & - & Identidad iberoamericana & - \\
\hline
\end{tabular}

«Decreto 89/2014, de 1 de agosto, por el que se establece la ordenación y el currículo de la Educación Primaria en la Comunidad Autónoma de Canarias». Boletín Oficial de Canarias, núm. 156.

\begin{tabular}{|l|c|l|c|}
\hline \multicolumn{2}{|c|}{ Ámbito europeo } & \multicolumn{2}{c|}{ Ámbito iberoamericano } \\
\hline Palabra clave & Frecuencia & Palabra clave & Frecuencia \\
\hline Europa & 19 & América & 6 \\
\hline Unión Europea & 15 & Iberoamérica & - \\
\hline Ciudadanía europea & - & Ciudadanía iberoamericana & - \\
\hline Identidad europea & - & Identidad iberoamericana & - \\
\hline
\end{tabular}

\begin{tabular}{|c|c|c|c|}
\hline \multicolumn{4}{|c|}{$\begin{array}{c}\text { «ecreto 27/2014, de } 5 \text { de junio, que establece el currículo de Educación } \\
\text { Primaria en la Comunidad Autónoma de Cantabria». Boletín Oficial de } \\
\text { Cantabria, núm. } 29 .\end{array}$} \\
\hline \multicolumn{2}{|c|}{ Ámbito europeo } & \multicolumn{2}{|c|}{ Ámbito iberoamericano } \\
\hline Palabra clave & Frecuencia & Palabra clave & Frecuencia \\
\hline Europa & 20 & América & 6 \\
\hline Unión Europea & 17 & Iberoamérica & - \\
\hline Ciudadanía europea & - & Ciudadanía iberoamericana & - \\
\hline Identidad europea & - & Identidad iberoamericana & - \\
\hline
\end{tabular}


«Decreto 54/2014, de 10/07/2014, por el que se establece el currículo de la Educación Primaria en la Comunidad Autónoma de Castilla-La Mancha». Diario Oficial de Castilla-La Mancha, núm. 132.

\begin{tabular}{|l|c|l|c|}
\hline \multicolumn{2}{|c|}{ Ámbito europeo } & \multicolumn{2}{c|}{ Ámbito iberoamericano } \\
\hline Palabra clave & Frecuencia & Palabra clave & Frecuencia \\
\hline Europa & 38 & América & 8 \\
\hline Unión Europea & 31 & Iberoamérica & - \\
\hline Ciudadanía europea & - & Ciudadanía iberoamericana & - \\
\hline Identidad europea & - & Identidad iberoamericana & - \\
\hline
\end{tabular}

«Decret 119/2015, de 23 de juny, d'ordenació dels ensenyaments de l'educació primària». Diari Oficial de la Generalitat de Catalunya, núm. 6900.

\begin{tabular}{|l|c|l|c|}
\hline \multicolumn{2}{|c|}{ Ámbito europeo } & \multicolumn{2}{c|}{ Ámbito iberoamericano } \\
\hline Palabra clave & Frecuencia & Palabra clave & Frecuencia \\
\hline Europa & 6 & América & - \\
\hline Unión Europea & 1 & Iberoamérica & - \\
\hline Ciudadanía europea & - & Ciudadanía iberoamericana & - \\
\hline Identidad europea & - & Identidad iberoamericana & - \\
\hline
\end{tabular}

«ORDEN EDU/519/2014, de 17 de junio, por la que se establece el currículo y se regula la implantación, evaluación y desarrollo de la educación primaria en la Comunidad de Castilla y León». Boletín Oficial de Castilla y León, núm. 117.

\begin{tabular}{|l|c|l|c|}
\hline \multicolumn{2}{|c|}{ Ámbito europeo } & \multicolumn{2}{c|}{ Ámbito iberoamericano } \\
\hline Palabra clave & Frecuencia & Palabra clave & Frecuencia \\
\hline Europa & 29 & América & 7 \\
\hline Unión Europea & 34 & Iberoamérica & - \\
\hline Ciudadanía europea & - & Ciudadanía iberoamericana & - \\
\hline Identidad europea & - & Identidad iberoamericana & - \\
\hline
\end{tabular}


«Decreto 103/2014, de 10 de junio, por el que se establece el currículo de Educación Primaria para la Comunidad Autónoma de Extremadura». Diario Oficial de Extremadura, núm. 114.

\begin{tabular}{|l|c|l|c|}
\hline \multicolumn{2}{|c|}{ Ámbito europeo } & \multicolumn{2}{c|}{ Ámbito iberoamericano } \\
\hline Palabra clave & Frecuencia & Palabra clave & Frecuencia \\
\hline Europa & 17 & América & 6 \\
\hline Unión Europea & 27 & Iberoamérica & - \\
\hline Ciudadanía europea & - & Ciudadanía iberoamericana & - \\
\hline Identidad europea & - & Identidad iberoamericana & - \\
\hline
\end{tabular}

«Decreto 105/2014, do 4 de setembro, polo que se establece o currículo da educación primaria na Comunidade Autónoma de Galicia». Diario Oficial de Galicia, núm. 171.

\begin{tabular}{|l|c|l|c|}
\hline \multicolumn{2}{|c|}{ Ámbito europeo } & \multicolumn{2}{c|}{ Ámbito iberoamericano } \\
\hline Palabra clave & Frecuencia & Palabra clave & Frecuencia \\
\hline Europa & 30 & América & 4 \\
\hline Unión Europea & 22 & Iberoamérica & - \\
\hline Ciudadanía europea & - & Ciudadanía iberoamericana & - \\
\hline Identidad europea & - & Identidad iberoamericana & - \\
\hline
\end{tabular}

\begin{tabular}{|c|c|c|c|}
\hline \multicolumn{4}{|c|}{$\begin{array}{c}\text { «Decreto 32/2014 de } 18 \text { de julio, por el que se establece el currículo de la } \\
\text { educación primaria en las Illes Balears». Butlletí Oficial de les Illes Balears, } \\
\text { núm. } 97 .\end{array}$} \\
\hline \multicolumn{2}{|c|}{ Ámbito europeo } & \multicolumn{2}{|c|}{ Ámbito iberoamericano } \\
\hline Palabra clave & Frecuencia & Palabra clave & Frecuencia \\
\hline Europa & 17 & América & 5 \\
\hline Unión Europea & 19 & Iberoamérica & - \\
\hline Ciudadanía europea & - & Ciudadanía iberoamericana & - \\
\hline Identidad europea & - & Identidad iberoamericana & - \\
\hline
\end{tabular}


«ecreto 24/2014, de 13 de junio, por el que se establece el currículo de la Educación Primaria en la Comunidad Autónoma de La Rioja». Boletín Oficial de La Rioja, núm. 74.

\begin{tabular}{|l|c|l|c|}
\hline \multicolumn{2}{|c|}{ Ámbito europeo } & \multicolumn{2}{c|}{ Ámbito iberoamericano } \\
\hline Palabra clave & Frecuencia & Palabra clave & Frecuencia \\
\hline Europa & 11 & América & 1 \\
\hline Unión Europea & 9 & Iberoamérica & - \\
\hline Ciudadanía europea & - & Ciudadanía iberoamericana & - \\
\hline Identidad europea & - & Identidad iberoamericana & - \\
\hline
\end{tabular}

«Decreto 89/2014, de 24 de julio, del Consejo de Gobierno, por el que se establece para la Comunidad de Madrid el Currículo de la Educación Primaria». Boletín Oficial de la Comunidad de Madrid, núm. 175.

\begin{tabular}{|l|c|l|c|}
\hline \multicolumn{2}{|c|}{ Ámbito europeo } & \multicolumn{2}{c|}{ Ámbito iberoamericano } \\
\hline Palabra clave & Frecuencia & Palabra clave & Frecuencia \\
\hline Europa & 11 & América & 4 \\
\hline Unión Europea & 7 & Iberoamérica & - \\
\hline Ciudadanía europea & - & Ciudadanía iberoamericana & - \\
\hline Identidad europea & - & Identidad iberoamericana & - \\
\hline
\end{tabular}

«Orden ECD/686/2014, de 23 de abril, por la que se establece el currículo de la Educación Primaria para el ámbito de gestión del Ministerio de Educación, Cultura y deporte y se regula su implantación, así como la evaluación y determinados aspectos organizativos de la etapa». Boletín Oficial del Estado, núm. 106

\begin{tabular}{|l|c|l|c|}
\hline \multicolumn{2}{|c|}{ Ámbito europeo } & \multicolumn{2}{c|}{ Ámbito iberoamericano } \\
\hline Palabra clave & Frecuencia & Palabra clave & Frecuencia \\
\hline Europa & 24 & América & 3 \\
\hline Unión Europea & 21 & Iberoamérica & - \\
\hline Ciudadanía europea & - & Ciudadanía iberoamericana & - \\
\hline Identidad europea & - & Identidad iberoamericana & - \\
\hline
\end{tabular}


«Decreto 198/2014, de 5 de septiembre, por el que se establece el currículo de la Educación Primaria en la Comunidad Autónoma de la Región de

Murcia». Boletín Oficial de la Región de Murcia, núm. 206.

\begin{tabular}{|l|c|l|c|}
\hline \multicolumn{2}{|c|}{ Ámbito europeo } & \multicolumn{2}{c|}{ Ámbito iberoamericano } \\
\hline Palabra clave & Frecuencia & Palabra clave & Frecuencia \\
\hline Europa & 11 & América & 7 \\
\hline Unión Europea & 18 & Iberoamérica & - \\
\hline Ciudadanía europea & - & Ciudadanía iberoamericana & - \\
\hline Identidad europea & - & Identidad iberoamericana & - \\
\hline
\end{tabular}

«Decreto Foral 60/2014, de 16 de julio, por el que se establece el currículo de las enseñanzas de Educación primaria en la Comunidad Foral de

Navarra». Boletín Oficial de Navarra, núm. 174.

\begin{tabular}{|l|c|l|c|}
\hline \multicolumn{2}{|c|}{ Ámbito europeo } & \multicolumn{2}{c|}{ Ámbito iberoamericano } \\
\hline Palabra clave & Frecuencia & Palabra clave & Frecuencia \\
\hline Europa & 26 & América & 3 \\
\hline Unión Europea & 19 & Iberoamérica & - \\
\hline Ciudadanía europea & - & Ciudadanía iberoamericana & - \\
\hline Identidad europea & - & Identidad iberoamericana & - \\
\hline
\end{tabular}

